\title{
Hipertensão Arterial entre Funcionários de Banco Estatal no Rio de Janeiro. Hábitos de Vida e Tratamento
}

\author{
Dóra Chor
}

Rio de Janeiro, RJ

\begin{abstract}
Objetivo - Estimar a freqüência de tratamento de hipertensão arterial e hábitos de vida relacionados à saúde, comparando-se hipertensos e não hipertensos.

Métodos - Estudo seccional em amostra sistemática de 1183 funcionários de banco estatal, no Rio de Janeiro, através de questionário autopreenchido. Medidas diretas da pressão arterial, peso e estatura foram tomadas em subamostra. Participantes informados por profissional de saúde, mais de uma vez, que eram hipertensos, foram classificados como hipertensos.

Resultados - Não houve diferença importante entre hipertensos e não hipertensos, quanto à prevalência de tabagismo, consumo de álcool e atividades fisicas. Entre os hipertensos com sobrepeso/obesidade, a prática de dieta foi mais freqüente do que entre não hipertensos com sobrepeso/obesidade. Apenas 44,7\% dos hipertensos estavam sob tratamento, condição associada à alta escolaridade, ser ex-fumante, ter sobrepeso/obesidade ou história familiar de doenças cerebrovasculares.

Conclusão - A disponibilidade de serviços de saúde e o acesso à informação não foram suficientes para garantir o tratamento ou adoção de hábitos de vida que contribuem para o controle da pressão arterial, no conjunto dos hipertensos.
\end{abstract}

Palavras-chave: hipertensão arterial, adesão ao tratamento, estilo de vida

\section{High Blood Pressure among Bank Employees. Life-Style and Treatment}

Purpose - This study estimates the frequency of treatment of high blood pressure and compares life-styles among hipertensives and non-hipertensives.

Methods-Cross-sectional study in a sistematic sample of 1183 employees in a government-owned bank in the State of Rio de Janeiro, through a self-administered questionnaire. Direct measurements of arterial pressure, weight and height were also taken in a sub-sample. Those who had been informed more than once as having high blood pressure, by a health professional, were classified as hipertensives.

Results - There were no important differences among hipertensives and non-hipertensives with respect to the prevalence of smoking, alcohol and physical activities. Dieting was more frequent among overweight/obese hipertensives than overweight/obese non-hipertensives. Only 44,7\% were under treatment. Subjects with high level of education were more likely to be treated as were those who quit smoking, presented overweight/obesity or family history of cerebrovascular diseases.

Conclusion - Access to information and health care was not sufficient to guarantee high blood pressure treatment or a healthy life-style which contributes to hypertension control.

Key-words: high blood pressure, treatment compliance, life style

Arq Bras Cardiol, volume $71\left(n^{0}\right.$ 5), 653-660, 1998

A hipertensão arterial (HA) tem sido indicada como o fator de risco conhecido de maior importância para a morbidade e mortalidade precoces causadas por doenças

Escola Nacional de Saúde Pública - Fundação Oswaldo Cruz - Rio de Janeiro Correspondência: Dóra Chor - Fundação Oswaldo Cruz - Depto de Epidemiologia - Rua Leopoldo Bulhões, 1480 - 21041-210 - Rio de Janeiro, RJ Recebido para publicação em 8/6/98 Aceito em 14/10/98 cardiovasculares. De fato, fortes evidências relacionam níveis elevados de pressão sistólica, diastólica ou ambas, com maior probabilidade de ocorrência de doença isquêmica do coração, doenças cerebrovasculares, aterosclerose e mortalidade geral ${ }^{1,2}$.

As estimativas de prevalência de HA, no Brasil, apresentam grande variação em função de diferentes critérios de classificação e instrumentos de medidas utilizados. Assim, 
os estudos populacionais disponíveis estimaram prevalências entre $15 \%$ e $30 \%$ para os homens e entre $15 \%$ e $27 \%$ para as mulheres ${ }^{3-5}$. Portanto, é uma enfermidade freqüente, cujos critérios para diagnóstico e recomendações para tratamento existem, e já foram aplicados, com sucesso, em programas dirigidos ao conjunto da população de outros países. Assim, por exemplo, a redução de $60 \%$ da mortalidade por doenças cerebrovasculares e de 53\% por doença coronariana, nos Estados Unidos, nos últimos 20 anos, tem sido associada ao aumento do número de hipertensos que tem conhecimento desta condição, encontram-se sob tratamento e apresentam níveis pressóricos controlados ${ }^{6}$. Outros autores sugerem que, além do tratamento farmacológico, a redução do hábito de fumar, mudanças do padrão de consumo alimentar e condições socioeconômicas também contribuíram para a redução da mortalidade, especialmente por doenças cerebrovasculares ${ }^{7}$.

A redução dos níveis tensionais do conjunto da população - hipertensos e não hipertensos - tem sido reconhecida como a estratégia potencialmente mais efetiva para o controle da HA, já que diminui a morbidade e a mortalidade cardiovascular, de forma tão ou mais expressiva do que o tratamento da enfermidade já estabelecida. Isto por que grande parte dos indivíduos que adoecem, especialmente no caso da doença coronariana, apresentam pressão arterial (PA) acima do que é considerado ótimo $(120 / 80 \mathrm{mmHg})$, sem no entanto atingirem níveis tensionais diagnosticados e tratados como HA. Além disso, o tratamento medicamentoso da doençajá estabelecida, nem sempre é seguido, implica em custose apresenta efeitos adversos. Assim, a redução dos níveis de PA no conjunto da população e a prevenção da doença estão baseadas, principalmente, no controle do peso, uso moderado de bebidas alcoólicas e de sal, prática regular de atividades físicas e consumo adequado de potássio através da dieta ${ }^{6}$.

Nossos resultados fazem parte de pesquisa sobre condições de saúde dos funcionários do Banco do Brasil, realizada em 1994. Os fatores de risco para doenças cardiovasculares foram investigados já que representam a principal causa de morte no Brasil e também neste grupo profissional, segundo estudo de suas causas de óbito, referente ao período entre $1977 \mathrm{e} 1990^{8}$. Neste artigo, apresentamos os resultados relativos ao tratamento da HA, bem como o perfil sóciodemográfico e de estilo de vida dos funcionários classificados como hipertensos, comparados aos não hipertensos.

\section{Métodos}

Realizou-se estudo seccional em amostra sistemática de 1183 funcionários do Banco do Brasil, lotados na Direção Geral e nos Centros de Processamento e Informação (Cesec), no Estado do Rio de Janeiro. Os dados foram coletados através de questionário autopreenchido no ambiente de trabalho, entre agosto e dezembro/94, onde diversas perguntas sobre a medida de PA e diagnóstico de HA foram incluídas, além de outros fatores de risco para doenças cardiovasculares.

Além do questionário, foram tomadas medidas diretas da PA, do peso e estatura de 322 funcionários, seleciona- dos aleatoriamente dentre os respondentes, a fim de comparar a classificação baseada nestas medidas com as respostas obtidas através do questionário ${ }^{9,10}$. Duas medidas de PA foram tomadas, uma antes e outra após o preenchimento do questionário, com estetoscópios e esfingmomanômetros de coluna de mercúrio. Tanto os aplicadores do questionário quanto os examinadores que tomaram as medidas diretas foram treinados e acompanhados por supervisão e controle de qualidade, de acordo com procedimentos padronizados ${ }^{11,12}$.

Os funcionários que declararam, no questionário, "terem sido informados, mais de uma vez, em dias diferentes, por um médico ou outro profissional de saúde, de que tinham HA ou pressão alta", foram classificados como hipertensos. Os funcionários submetidos à medida direta da PA foram classificados como hipertensos quando em uso de medicamento específico e/ou quando a $2^{\mathrm{a}}$ medida da PA foi $\geq 160 / 95 \mathrm{mmHg}{ }^{13}$. Foram considerados "em tratamento" aqueles que declararam estar em uso de medicamentos específicos, ou em dieta com restrição de sal ou para perda de peso, ou que praticavam atividades físicas para controle dos níveis tensionais.

Características sócio-demográficas e de estilo de vida dos funcionários classificados como hipertensos e não hipertensos foram estudadas: fumo, consumo de álcool (diário, semanal, ocasional ou nunca), atividade física de lazer (praticar pelo menos duas vezes por semana, durante 20min cada vez) e estar sob dieta (para emagrecer, com restrição de açúcar, com restrição de gordura). Os funcionários informaram seu peso e estatura no questionário, e a partir dessas variáveis calculou-se o índice de massa corporal (IMC) (peso em kg/estatura ${ }^{2} \mathrm{em}$ metros). Aqueles funcionários cujos IMC situaram-se acima de $25 \mathrm{~kg} / \mathrm{m}^{2}$ foram classificados como apresentando sobrepeso ou obesidade ${ }^{14}$.

Em função das diferentes frações amostrais dos dois estratos (Cesec e Direção Geral), os estimadores foram corrigidos por um fator de expansão, constituído pelo inverso da fração amostral de cada estrato, multiplicada pela razão entre o total da amostra (1183) e a população de estudo (4457).

A validade das informações contidas no questionário a respeito da HA e do IMC foi avaliada por meio da análise de sensibilidade, especificidade e valores preditivos ${ }^{15}$. Além disso, a concordância entre as duas fontes de informação foi medida através do coeficiente Kappa ${ }^{16} \mathrm{e}$, no caso do peso e do IMC, também através do coeficiente de correlação intraclasse ${ }^{10,17}$.

Diferenças entre proporções foram testadas com o teste do qui-quadrado. Variáveis contínuas foram categorizadas em tercis ou quintis. Ao analisar a situação conjugal, os funcionários casados, separados e viúvos foram agrupados na mesma categoria em função do pequeno número de separados e viúvos, e por apresentarem características semelhantes em relação às variáveis de interesse. Razões de chance (odds ratios $(\mathrm{OR})$ ) ajustadas por sexo e idade foram obtidas através da análise de regressão logística, e seus intervalos de confiança através da exponenciação dos respectivos coeficientes de regressão e erros-padrão. Todas as análises foram realizadas através do software SPSS for Windows versão 6.1 ${ }^{18}$. 


\section{Resultados}

A adesão ao estudo foi bastante elevada já que $93 \%$ dos funcionários sorteados responderam ao questionários. Dentre esses, $60 \%$ eram homens e $40 \%$ mulheres, com idade média de 37,4 anos e 39,1 anos, respectivamente ( $\mathrm{p}<0,001)$. Trata-se portanto de uma população jovem, com alto nível de escolaridade - cerca de $90 \%$ dos homens e das mulheres apresentavam curso superior completo ou incompleto (tab. I).

Uma vez que a medida de $\mathrm{PA}$ pertencia à rotina do exame de saúde realizado anualmente, em todos os funcionários, cerca de $100 \%$ informaram que tiveram seus níveis tensionais medidos nos últimos dois anos antes da pesquisa. Mesmo assim, 29,5\% dos homens não souberam informar seus níveis habituais de PA, comparados a $12,8 \%$ das mulheres.

A validade da classificação de HA baseada na informação contida no questionário foi avaliada através da comparação com a medida direta (anexo 1). O valor preditivo positivo da informação foi bastante elevado, variando entre 73,3\% (Direção Geral)e 100\% (agência Ilha do Governador). Omesmo ocorreu em relação ao valor preditivo negativo, que variou entre $91,2 \%$ no Cesec e $84 \%$ na direção geral. A especificidade da informação foi também bastante elevada, variando entre $100 \%$ na agência Ilha do Governador e 95,2\% na Direção Geral. A sensibilidade, por outro lado, foi moderada, situando-se entre 42,3\% na Direção Geral e 58,3\% no Cesec.

\begin{tabular}{|c|c|c|c|c|}
\hline \multicolumn{5}{|c|}{$\begin{array}{l}\text { Tabela I - Distribuição dos funcionários que participaram do } \\
\text { estudo segundo sexo, idade e escolaridade }\end{array}$} \\
\hline \multirow[t]{2}{*}{ Variáveis } & \multicolumn{2}{|c|}{ Homens } & \multicolumn{2}{|c|}{ Mulheres } \\
\hline & $\mathrm{n}$ & $\% *$ & $\mathrm{n}$ & $\% *$ \\
\hline Sexo & 689 & 60,0 & 494 & 40,0 \\
\hline \multicolumn{5}{|l|}{ Idade } \\
\hline $20-34$ & 226 & 34,8 & 85 & 17,4 \\
\hline $35-39$ & 184 & 26,0 & 203 & 39,5 \\
\hline $40-44$ & 196 & 28,2 & 161 & 34,2 \\
\hline 45 e mais & 83 & 10,9 & 45 & 8,9 \\
\hline \multicolumn{5}{|l|}{ Escolaridade } \\
\hline $2^{\circ}$ grau completo/incompleto & 72 & 12,1 & 43 & 10,3 \\
\hline Superior incompleto & 234 & 36,0 & 97 & 19,9 \\
\hline Superior completo & 383 & 51,9 & 354 & 69,7 \\
\hline
\end{tabular}

Anexo 1

Comparação entre a classificação de hipertensão arterial segundo medida e informação do questionário Agência Centro

\begin{tabular}{|c|c|c|c|c|c|c|c|}
\hline \multirow{3}{*}{$\begin{array}{l}\text { Medida ou } \\
\text { Medicamento }\end{array}$} & \multicolumn{7}{|c|}{ Questionário } \\
\hline & \multicolumn{3}{|c|}{ Não hipertenso } & \multicolumn{3}{|c|}{ Hipertenso } & \multirow{2}{*}{$\begin{array}{l}\text { Total } \\
\mathrm{n} \%\end{array}$} \\
\hline & $\mathrm{n}$ & $\% *$ & $\% * *$ & $\mathrm{n}$ & $\% *$ & $\% * *$ & \\
\hline Não hipertenso & 65 & 98,5 & 86,7 & 1 & 1,5 & 10,0 & 66100,0 \\
\hline Hipertenso & 10 & 52,6 & 13,3 & 9 & 47,4 & 90,0 & 19100,0 \\
\hline Total & 75 & 88,2 & 100,0 & 10 & 11,8 & 100,0 & 85100,0 \\
\hline
\end{tabular}

Comparação entre a classificação de hipertensão arterial segundo medida e informação do questionário

\section{Cesec}

\begin{tabular}{|c|c|c|c|c|c|c|c|c|}
\hline \multirow{3}{*}{$\begin{array}{l}\text { Medida ou } \\
\text { Medicamento }\end{array}$} & \multicolumn{8}{|c|}{ Questionário } \\
\hline & \multicolumn{3}{|c|}{ Não hipertenso } & \multicolumn{3}{|c|}{ Hipertenso } & \multicolumn{2}{|c|}{ Total } \\
\hline & $\mathrm{n}$ & $\% *$ & $\%$ ** & $\mathrm{n}$ & $\% *$ & $\% * *$ & & $\%$ \\
\hline Não hipertenso & 52 & 98,1 & 91,2 & 1 & 1,9 & 12,5 & 53 & 100,0 \\
\hline Hipertenso & 5 & 41,7 & 8,8 & 7 & 58,3 & 87,5 & 12 & 100,0 \\
\hline Total & 57 & 87,7 & 100,0 & 8 & 12,3 & 100,0 & 65 & 100,0 \\
\hline
\end{tabular}

Comparação entre a classificação de hipertensão arterial segundo medida e informação do questionário

\section{Direção Geral}

\begin{tabular}{|lccccccccc}
\hline & \multicolumn{1}{c|}{ Questionário } \\
\cline { 2 - 10 } Medida ou & \multicolumn{1}{c}{ Não hipertenso } & \multicolumn{3}{c}{ Hipertenso } & \multicolumn{2}{c}{ Total } \\
Medicamento & $\mathrm{n}$ & $\%^{*}$ & $\%^{* *}$ & $\mathrm{n}$ & $\%^{*}$ & $\%{ }^{* *}$ & $\mathrm{n}$ & $\%$ \\
\hline Não hipertenso & 79 & 95,2 & 84,0 & 4 & 4,8 & 26,7 & 83 & 100,0 \\
Hipertenso & 15 & 57,7 & 16,0 & 11 & 42,3 & 73,3 & 26 & 100,0 \\
Total & 94 & 86,2 & 100,0 & 15 & 13,8 & 100,0 & 109 & 100,0 \\
\hline
\end{tabular}

Kappa $=0,44 \mathrm{p}<0,001 ; *$ percentual na linha; $* *$ percentual na coluna.

Comparação entre a classificação de hipertensão arterial segundo medida e informação do questionário

\section{Ilha do Governador}

\begin{tabular}{|lccccccccc|}
\hline & \multicolumn{1}{c|}{ Questionário } \\
\cline { 2 - 9 } Medida ou & \multicolumn{1}{c|}{ Não Hipertenso } & \multicolumn{4}{c|}{ Hipertenso } & \multicolumn{2}{l}{ Total } \\
Medicamento & $\mathrm{n}$ & $\%^{*}$ & $\%^{* *}$ & $\mathrm{n}$ & $\%^{*}$ & $\%^{* *}$ & $\mathrm{n}$ & $\%$ \\
\hline Não hipertenso & 40 & 100,0 & 88,9 & 0 & - & - & 40 & 100,0 \\
Hipertenso & 5 & 45,5 & 11,1 & 6 & 54,5 & 100,0 & 11 & 100,0 \\
Total & 45 & 88,2 & 100,0 & 6 & 11,8 & 100,0 & 51 & 100,0 \\
\hline Kappa $=0,65 \mathrm{p}<0,001 ; *$ percentual na linha; & $* *$ percentual na coluna. \\
\hline
\end{tabular}

Quanto à validade do IMC baseada na informação de peso e estatura contidas no questionário, a sensibilidade variou entre 76,2\% no Cesec e $96 \%$ na agência Centro, enquanto a especificidade foi próxima a $100 \%$ em todas as dependências (anexo 2). Os valores preditivos positivo e negativo foram também bastante elevados, situando-se acima de $88 \%$ (anexo 2). Quanto à confiabilidade, o coeficiente de correlação intraclasse variou entre 0,984 e 0,992 no caso do peso, e entre 0,949 e 0,984 no caso do IMC $^{10}$. As diferenças entre a informação e medida da estatura foram consideradas irrelevantes ${ }^{10}$. De acordo com estes resultados, o IMC seria classificado corretamente a partir das informações contidas no questionário, na grande maioria dos casos.

A prevalência de HA foi estimadaem 18,3\%, comintervalo de $95 \%$ de confiança (IC 95\%) entre $14,6 \%$ e $22,0 \%$. A 


\section{Anexo 2}

Comparação entre a classificação do índice de massa corporal segundo medida e informação do questionário

\section{Agência centro}

\begin{tabular}{|c|c|c|c|c|c|c|c|}
\hline \multirow[b]{3}{*}{ Medida } & \multicolumn{7}{|c|}{ Questionário } \\
\hline & \multicolumn{3}{|c|}{$\begin{array}{c}\text { Normal e } \\
\text { baixo peso }\end{array}$} & \multicolumn{2}{|c|}{$\begin{array}{l}\text { Soprepeso e } \\
\text { obesidade }\end{array}$} & \multicolumn{2}{|c|}{ Total } \\
\hline & $\mathrm{n}$ & $\% *$ & $\% * *$ & $\mathrm{n} \quad \%^{*}$ & $\% * *$ & $\mathrm{n}$ & $\%$ \\
\hline Normal e baixo peso & 51 & 98,1 & 98,1 & $1 \quad 1,9$ & 4,0 & 52 & 100,0 \\
\hline Sobrepeso e obesidade & 1 & 4,0 & 4,0 & 2496,0 & 96,0 & 25 & 100,0 \\
\hline Total & 52 & 67,5 & 100,0 & 2532,5 & 100,0 & 77 & 100,0 \\
\hline
\end{tabular}

Kappa $=0,94 \mathrm{p}<0,001 ; *$ percentual na linha; $* *$ percentual na coluna

Comparação entre a classificação do índice de massa corporal segundo medida e informação do questionário

Cesec

\begin{tabular}{|c|c|c|c|c|c|c|c|c|}
\hline \multirow[b]{3}{*}{ Medida } & \multicolumn{8}{|c|}{ Questionário } \\
\hline & \multicolumn{3}{|c|}{$\begin{array}{l}\text { Normal e } \\
\text { baixo peso }\end{array}$} & \multicolumn{3}{|c|}{$\begin{array}{l}\text { Soprepeso e } \\
\text { obesidade }\end{array}$} & \multicolumn{2}{|c|}{ Total } \\
\hline & $\mathrm{n}$ & $\% *$ & $\%$ ** & $\mathrm{n}$ & $\% *$ & $\% * *$ & $\mathrm{n}$ & $\%$ \\
\hline Normal e baixo peso & 37 & 94,9 & 88,1 & 2 & 5,1 & 11,1 & 39 & 100,0 \\
\hline Sobrepeso e obesidade & 5 & 23,8 & 11,9 & 16 & 76,2 & 88,9 & 21 & 100,0 \\
\hline Total & 42 & 70,0 & 100,0 & 18 & 30,0 & 100,0 & 60 & 100,0 \\
\hline
\end{tabular}

Comparação entre a classificação do índice de massa corporal segundo medida e informação do questionário

\section{Direção Geral}

\begin{tabular}{|c|c|c|c|c|c|c|c|c|}
\hline \multirow[b]{3}{*}{ Medida } & \multicolumn{8}{|c|}{ Questionário } \\
\hline & \multicolumn{3}{|c|}{$\begin{array}{c}\text { Normal e } \\
\text { baixo peso }\end{array}$} & \multicolumn{3}{|c|}{$\begin{array}{l}\text { Soprepeso e } \\
\text { obesidade }\end{array}$} & \multicolumn{2}{|c|}{ Total } \\
\hline & $\mathrm{n}$ & $\% *$ & $\% * *$ & $\mathrm{n}$ & $\%^{*}$ & $\% * *$ & $\mathrm{n}$ & $\%$ \\
\hline Normal e baixo peso & 67 & 97,1 & 93,1 & 2 & 2,9 & 5,7 & 69 & 100,0 \\
\hline Sobrepeso e obesidade & 5 & 13,2 & 6,9 & 33 & 86,8 & 94,3 & 38 & 100,0 \\
\hline Total & 72 & 67,3 & 100,0 & 35 & 32,7 & 100,0 & 107 & 100,0 \\
\hline
\end{tabular}

Comparação entre a classificação do índice de massa corporal segundo medida e informação do questionário

\section{Ilha do Governador}

\begin{tabular}{|c|c|c|c|c|c|c|c|}
\hline \multirow[b]{3}{*}{ Medida } & \multicolumn{7}{|c|}{ Questionário } \\
\hline & \multicolumn{3}{|c|}{$\begin{array}{l}\text { Normal e } \\
\text { baixo peso }\end{array}$} & \multicolumn{2}{|c|}{$\begin{array}{l}\text { Sobrepso e } \\
\text { obesidade }\end{array}$} & \multicolumn{2}{|c|}{ Total } \\
\hline & $\mathrm{n}$ & $\% *$ & $\% * *$ & $\mathrm{n} \quad \% *$ & $\% * *$ & $\mathrm{n}$ & $\%$ \\
\hline Normal e baixo peso & 28 & 96,6 & 93,3 & 3,4 & 8,3 & 29 & 100,0 \\
\hline Sobrepeso e obesidade & 2 & 15,4 & 6,7 & 1184,6 & 91,7 & 13 & 100,0 \\
\hline Total & 30 & 71,4 & 100,0 & 1228,6 & 100,0 & 42 & 100,0 \\
\hline
\end{tabular}

prevalência foi maior entre os homens $(22,3 \%)$ do que entre as mulheres $(12,6 \%)(\mathrm{p}<0,001)$. Além disso, a prevalência aumentou com a idade: $14,2 \%$ dos funcionários $<40$ anos e $24,5 \%$ dos $>40$ anos foram classificados como hipertensos $(\mathrm{p}<0,001)$.

Não se observaram diferenças significativas entre hipertensos e não hipertensos quanto à prevalência de consumo de álcool e sedentarismo, embora maior proporção de hipertensos tenha declarado consumir álcool diariamente, em ambos os grupos etários analisados (tab. II). A prevalência de tabagismo, por outro lado, variou em função da idade, entre hipertensos e não hipertensos. Entre os mais jovens, maior proporção de hipertensos fumavam (34,1\%; IC 95\%: 27,3-40,9), comparados aos não hipertensos (22,3\%; IC 95\%: 17,8-26,8). Além disso, a prevalência de ex-fumantes foi bem maior entre os hipertensos, sugerindo que o diagnóstico da doença pode estar relacionado ao abandono do hábito de fumar. Entre os funcionários com mais de 35 anos, não houve diferença importante quanto ao hábito de fumar, comparando-se hipertensos e não hipertensos.

\begin{tabular}{|c|c|c|c|c|}
\hline \multicolumn{5}{|c|}{$\begin{array}{c}\text { Tabela II - Distribuição do consumo de álcool, atividades físicas de } \\
\text { lazer e hábito de fumar entre hipertensos e não hipertensos } \\
\text { por grupo etário }\end{array}$} \\
\hline \multirow[t]{3}{*}{ Hábitos } & \multirow{2}{*}{\multicolumn{2}{|c|}{$\begin{array}{c}\text { Menores de } 35 \text { anos (\%*) } \\
\text { Não }\end{array}$}} & \multirow{3}{*}{\multicolumn{2}{|c|}{$\begin{array}{c}\text { Maiores de } 35 \operatorname{anos}(\% *) \\
\text { Não } \\
\text { Hipertensos Hipertenso }\end{array}$}} \\
\hline & & & & \\
\hline & Hipertensos & Hipertensos & & \\
\hline \multicolumn{5}{|c|}{ Consumo de álcool (a) } \\
\hline Diário & 11,4 & 6,7 & 14,5 & 10,2 \\
\hline Semanal & 47,7 & 40,4 & 32,0 & 31,4 \\
\hline Ocasional & 31,8 & 44,3 & 43,0 & 50,3 \\
\hline Nunca & 9,1 & 8,5 & 10,5 & 8,1 \\
\hline \multicolumn{5}{|c|}{$\begin{array}{l}\text { Atividades físicas } \\
\text { de lazer (b) }\end{array}$} \\
\hline Praticam & 45,5 & 48,9 & 39,0 & 41,0 \\
\hline \multicolumn{5}{|l|}{ Tabagismo (c) } \\
\hline Fumante atual & 34,1 & 22,3 & 35,8 & 33,5 \\
\hline Ex-fumante & 20,5 & 8,2 & 27,2 & 24,5 \\
\hline Nunca fumou & 45,5 & 69,5 & 37,0 & 42,0 \\
\hline Total** & 207 & 967 & 207 & 967 \\
\hline \multicolumn{5}{|c|}{$\begin{array}{l}\text { *Proporções corrigidas por fator de expansão (vide metodologia); **9 funcio- } \\
\text { nários não responderam a pergunta utilizada para classificação de hipertensão } \\
\text { arterial; a: }<35 \text { anos- } \mathrm{p}=0,3904 ;>35 \text { anos- } \mathrm{p}=0,19656 ; \mathrm{b}:<35 \text { anos-p=0,66653; } \\
>35 \text { anos-p=0,61940; c: }<35 \text { anos- } \mathrm{p}=0,00337 ;>35 \text { anos- } \mathrm{p}=0,48377 \text {. }\end{array}$} \\
\hline
\end{tabular}

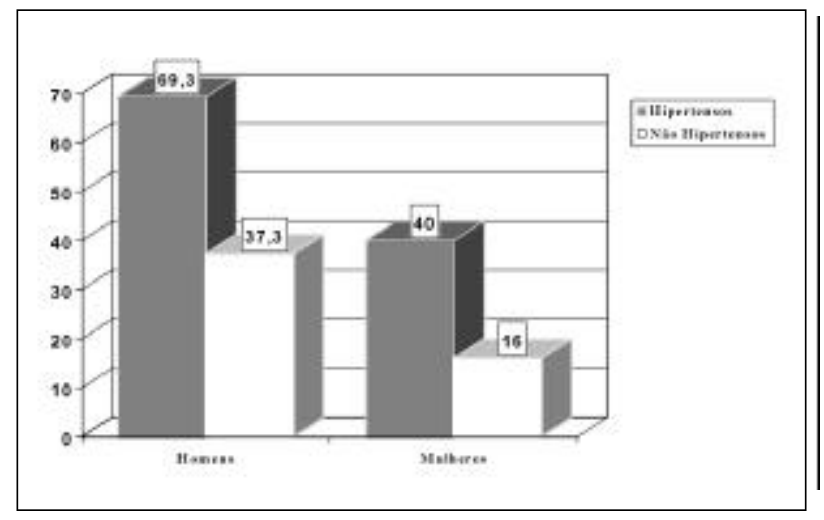

Gráfico 1 - Prevalência de sobrepeso/obesidade entre hipertensos e não hipertensos segundo sexo 
Já que a obesidade é um dos principais fatores de risco para a HA, observamos alta proporção de sobrepeso e obesidade entre os homens hipertensos: $69,3 \%$ apresentaram IMC acima do normal, comparados a 37,3\% dos não hipertensos (gráfico 1). Entre as funcionárias hipertensas, alta prevalência de sobrepeso/obesidade também foi estimada (40\%), comparada a $16 \%$ entre as não hipertensas. Apesar da alta prevalência de sobrepeso e obesidade entre os hipertensos, a prática de dieta para emagrecer e dieta com restrição de açúcar ou gorduras foi mais freqüente neste grupo, quando comparado aos não hipertensos com sobrepeso ou obesidade (tab. III). De fato, dentre os comportamentos estudados, a prática de dieta foi o único que apresentou associação evidente com o diagnóstico de HA.

Menos da metade dos hipertensos $-44,7 \%$ - (IC 95\%: 40\%-49,4\%) declarou estar sob tratamento específico, medicamentoso ou não (dieta para perder peso, dieta com restrição de sal ou prática de atividades físicas). A proporção de mulheres em tratamento foi maior do que de homens - $50 \% \mathrm{e}$ $43 \%$ - respectivamente, embora sem diferença estatisticamente significativa $(\mathrm{p}=0,48)(\mathrm{tab}$. IV). Quanto aos hipertensos que não se encontravam sob tratamento, mais de um quinto nunca se tratou. Entre aqueles que interromperam o tratamento, $46,3 \%$ justificaram esta decisão por "terem ficado curados".

A parcela de hipertensos que declarou estar sob tratamento específico variou de forma importante com a idade (tab. V): apenas $17,1 \%$ dos mais jovens se tratavam, chegando esta proporção a 77,4\% entre os de 45 anos ou mais. Os medicamentos e a dieta com restrição de sal foram as modalidades de tratamento mais utilizados $(81 \%$ e $75 \%$, respectivamente), enquanto $48 \%$ faziam dieta para perder peso e $36 \%$ praticavam atividades físicas.

\begin{tabular}{|c|c|c|c|c|}
\hline \multicolumn{5}{|c|}{$\begin{array}{l}\text { Tabela III - Prática de dieta entre funcionários com sobrepeso ou } \\
\text { obesidade* segundo classificação de hipertensão arterial }\end{array}$} \\
\hline \multirow[t]{2}{*}{ Tipo** } & \multicolumn{2}{|c|}{ Hipertensos } & \multicolumn{2}{|c|}{ Não hipertensos } \\
\hline & $\mathrm{n}$ & $\% * * *$ & $\mathrm{n}$ & $\% * * *$ \\
\hline Dieta para emagrecer (a) & 28 & 36,0 & 53 & 19,7 \\
\hline Dieta com restrição de açúcar (b) & 20 & 24,7 & 42 & 14,6 \\
\hline Dieta com restrição de gorduras (c) & 22 & 27,3 & 48 & 17,3 \\
\hline \multicolumn{5}{|c|}{$\begin{array}{l}\text { *Índice de massa corporal }>25 \mathrm{~kg} / \mathrm{m}^{2} ; * * \text { mais de um tipo de dieta podia ser } \\
\text { referido por cada funcionário; *** proporções corrigidas por fator de expan- } \\
\text { são (vide metodologia); a- } \mathrm{p}<0,001 ; \mathrm{b}-\mathrm{p}=0,02625 ; \mathrm{p}=0,03974 \text {. }\end{array}$} \\
\hline
\end{tabular}

\begin{tabular}{|lcc|}
\hline $\begin{array}{l}\text { Tabela IV - Condição de tratamento para o controle de hiperten- } \\
\text { são arterial segundo sexo }\end{array}$ \\
\hline Condição de tratamento* & $\begin{array}{c}\text { Homens } \\
\%\end{array}$ & $\begin{array}{c}\text { Mulheres } \\
\%\end{array}$ \\
\hline Trataram no passado & 26,8 & 22,9 \\
Estão em tratamento & 43,0 & 50,0 \\
Nunca se trataram & 30,2 & 27,1 \\
Total & 148 & 59 \\
\hline Proporções corrigidas por fator de expansão (vide metodologia); *não \\
houve diferença estatisticamente significativa entre os sexos (p=0,69075). \\
\hline
\end{tabular}

\begin{tabular}{|lcc|}
\hline \multicolumn{3}{|c|}{$\begin{array}{c}\text { Tabela V - Distribuição dos hipertensos sob tratamento para o } \\
\text { controle de hipertensão arterial segundo idade }\end{array}$} \\
\hline Idade & Proporção em tratamento & Total \\
& $\%^{*}$ & \\
\hline $20-34$ & 17,1 & 37 \\
$35-39$ & 33,3 & 50 \\
$40-44$ & 54,1 & 69 \\
45 e mais & 77,4 & 32 \\
\hline *Proporções corrigidas por fator de expansão (vide metodologia); $\chi^{2}$ \\
tendência linear: $\mathrm{p}<0,001$. \\
\hline
\end{tabular}

\begin{tabular}{|c|c|c|}
\hline \multicolumn{3}{|c|}{$\begin{array}{l}\text { Tabela VI - Associação* entre tratamento de hipertensão arterial e } \\
\text { características sócio-demográficas e de estilo de vida }\end{array}$} \\
\hline Característica & OR & $\begin{array}{l}\text { Intervalo de } \\
\text { confiança }\end{array}$ \\
\hline Escolaridade $\left(>2^{\circ}\right.$ grau $x \leq 2^{\circ}$ grau $)$ & 6,22 & $1,76-21,97$ \\
\hline Renda per capita & & \\
\hline Até $\mathrm{R} \$ 400,00 \mathrm{x}>\mathrm{R} \$ 666,67$ & 1,03 & $0,44-2,40$ \\
\hline Entre $R \$ 400,00$ e $R \$ 666,67 x>R \$ 666,67$ & 0,42 & $0,17-1,03$ \\
\hline $\begin{array}{l}\text { Situação conjugal } \\
\text { Casados/separados/viúvos x solteiros }\end{array}$ & 2,02 & $0,93-4,35$ \\
\hline Tabagismo & & \\
\hline Ex-fumante $\mathrm{x}$ fumante atual & 2,13 & $0,97-4,73$ \\
\hline Ex-fumante $\mathrm{x}$ nunca fumou & 2,63 & $1,18-6,01$ \\
\hline Consumo de álcool & & \\
\hline Ocasional/nunca x semanal/diário & 1,29 & $0,69-2,43$ \\
\hline Índice de massa corporal & & \\
\hline Sobrepeso/obesidade x normal/baixo peso & 2,32 & $1,12-4,81$ \\
\hline História familiar (parentes consangüíneos) & & \\
\hline Hipertensão arterial (sim x não) & 0,81 & $0,39-1,69$ \\
\hline Doenças cerebrovasculares (sim x não) & 2,50 & $1,30-4,82$ \\
\hline Infarto agudo do miocárdio (sim x não) & 0,83 & $0,44-1,56$ \\
\hline
\end{tabular}

A associação entre HA sob tratamento e variáveis sócio-demográficas e de estilo de vida é apresentada na tabela VI através de razões de chance (OR) ajustadas por idade e sexo. A escolaridade apresentou-se fortemente associada ao tratamento: os que tinham curso superior completo ou incompleto apresentaram chance cerca de seis vezes maior de se tratar do que os que não chegaram a ingressar na universidade.

A associação com a renda per capita revelou-se inconsistente. As proporções de funcionários em tratamento no tercil de menor renda (até $\mathrm{R} \$ 400,00$ ) e no tercil de maior ren$\mathrm{da}(>\mathrm{R} \$ 666,67)$ foram semelhantes. Por outro lado, aqueles situados na faixa intermediária de renda per capita apresentaram menor prevalência de tratamento.

A situação conjugal também se apresentou fortemente associada à condição de tratamento, embora sua significância estatística tenha sido marginal. Os hipertensos casados, separados ou viúvos, apresentaram chance duas vezes maior de estarem sob tratamento comparados aos solteiros.

Os hipertensos que abandonaram o hábito de fumar apresentaram a maior prevalência de tratamento - mais de duas vezes maior do que os que nunca fumaram e também 
dos que fumavam à época da pesquisa - assim como os que consumiam bebida alcoólica ocasionalmente ou nunca, embora esta associação não tenha sido estatisticamente significativa.

O IMC apresentou forte associação com o tratamento: os hipertensos com sobrepeso ou obesidade tiveram chance cerca de duas vezes maior de estarem em tratamento.

Em relação à história familiar, somente os que tinham parentes com doenças cerebrovasculares apresentaram associação positiva com o tratamento ( 2,5 vezes maior). História familiar de HA e de infarto agudo do miocárdio (IAM), por outro lado, diminuíram a chance de estar em tratamento, embora os resultados não tenham alcançado significância estatística.

\section{Discussão}

As doenças cardiovasculares continuam sendo a principal causa de morte no Brasil. Três de seus principais fatores de risco são passíveis de modificação - fumo, HA e hipercolesterolemia -e, portanto, a redução de sua prevalência tem sido considerada prioritária nos países desenvolvidos ${ }^{19,20}$.

Ainda não se conhece o impacto real da HA na mortalidade brasileira em função da tabulação exclusiva da causa básica do óbito, onde esta enfermidade é raramente mencionada. Novas investigações serão possíveis a partir do estudo das causas múltiplas de óbito, hoje realizado em poucas regiões do país. Estudos de prevalência, por outro lado, já têm indicado a alta magnitude da hipertensão em várias cidades brasileiras, especialmente, entre a população mais pobre $^{3,21,22}$, acrescentando, assim, importante risco adicional à saúde deste grupo, que já apresenta piores condições de vida e trabalho, maior nível de desemprego e maiores dificuldades de acesso aos serviços de saúde. Já é possível, portanto, mencionar a hipertensão como uma das principais doenças crônicas não transmissíveis no Brasil, em termos de magnitude e conseqüências graves. Este destaque é ainda mais importante na população entre 35 e 54 anos, cuja prevalência de obesidade - um dos principais fatores de risco para a hipertensão - vem aumentando ${ }^{23}$.

Em nossos resultados, a prevalência de HA foi compatível com aquelas estimadas em outros estudos nacionais, que utilizaram a medida direta para a classificação da população, levando-se em conta diferenças de distribuição etária e de nível social dos grupos investigados ${ }^{3,4,24}$.

A confiabilidade da pergunta utilizada para a classificação de hipertensão "Algum médico ou outro profissional de saúde já lhe informou que você tinha HA ou pressão alta?" foi alta, estimada em 0,82 (coeficiente kappa), no "Missouri Behavioral Risk Factor Survey" ${ }^{25}$. No caso dessa investigação, variou entre 0,44 e 0,65 , nas quatro unidade do banco onde a medida direta foi realizada (anexo 1), valores que podem ser considerados entre moderados a bons ${ }^{16}$.

Em estudos estrangeiros, a sensibilidade da pergunta foi bastante elevada, 84\% nos Estados Unidos e 74\% no Canadá ${ }^{26,27}$. Em Porto Alegre, 57,7\% dos indivíduos classificados como hipertensos conheciam sua condição ${ }^{28}$. No caso dos bancários, a classificação baseada no questionário identificou corretamente os normotensos (especificidades e valores preditivos negativos elevados - acima de $90 \%$ ). A maior parte dos funcionários classificados como hipertensos através do questionário foi também confirmada pela medida (valores preditivos positivos acima de $73 \%$ ). A fragilidade do questionário encontra-se, portanto, na proporção de falso-negativos, em função dos valores moderados de sensibilidade. Essa fragilidade não comprometeu, provavelmente, os resultados encontrados, admitindo-se que a prevalência de hipertensão, baseada no questionário, está subestimada, mas que a chance de cometer erro inverso, classificando não hipertensos como hipertensos, foi bem menor, e ainda que os erros de classificação existentes se distribuíram, provavelmente, de maneira não diferenciada entre as categorias de outros fatores de risco (hipertensos fumantes ou não fumantes, sedentários ou não). Embora reconhecendo a importância desta limitação dos dados, nosso objetivo principal foi o de caracterizar comportamentos e aderência ao tratamento daqueles funcionários "informados mais de uma vez de que eram hipertensos", e que, portanto, deveriam apresentar estilo de vida adequado ao controle da PA. Tendo em vista este objetivo, a estimativa da prevalência de HA, bem como a comparação com a medida direta, tornam-se secundários.

A rigor, estudos de validação do tipo que realizamos podem estar comparando coisas distintas, que têm objetivos diferentes: classificação de um grupo populacional para estimativa de prevalência e diagnóstico clínico de HA. Em função dessas diferenças, discordâncias entre as duas fontes de informação podem ser atribuídas, por exemplo, aos diferentes pontos de corte utilizados para a classificação em pesquisa - onde inúmeros autores ${ }^{3,5,28,30}$ também seguiram o critério OMS ${ }^{13}$ de 160/95mmHg - ou para o diagnóstico clínico (140/90mmHg). O critério adotado (160/ $95 \mathrm{mmHg}$ ) diminui o número de falso-positivos já que uma ou mais medidas da PA são tomadas em um mesmo dia, e que elevações transitórias da PA, entre 140/90mmHg e 160/ $95 \mathrm{mmHg}$, são freqüentes. Outras justificativas para as discrepâncias entre o questionário e a medida direta dizem respeito à ausência do diagnóstico médico, aos diferentes critérios adotados pelos médicos para o diagnóstico e, nos casos em que os níveis tensionais não são muito altos - a maioria - a doença pode ser diagnosticada por alguns e negada por outros, deixando o indivíduo em dúvida sobre sua condição.

Considerando-se o alto nível de escolaridade e o amplo acesso aos Serviços de Saúde através de unidades credenciadas, serviços próprios, e ainda do reembolso de despesas, os resultados relativos à proporção de funcionários hipertensos em tratamento e ao estilo de vida não eram esperados. Apenas $43 \%$ dos homens e $50 \%$ das mulheres estavam em tratamento, comparados por exemplo a $84 \%$ e $90 \%$ de homens e mulheres, respectivamente, estudados em amostra da população norte-americana no "Atherosclerosis Risk in Communities" (ARIC) ${ }^{26}$. Em nove províncias canadenses, esta proporção foi de $86 \%$ dos hipertensos, considerando-se ambos os sexos ${ }^{27}$. 
As características associadas ao tratamento variaram conforme a população estudada. No caso dos bancários, embora as mulheres conhecessem seus níveis habituais de PA com maior freqüência, não houve diferença significativa, entre os sexos, da prevalência de tratamento, enquanto maior aderência entre as mulheres tem sido relatada ${ }^{27,28}$. Quanto à idade, alguns estudos não encontraram associação ${ }^{26,29}$, enquanto outros confirmaram os resultados desta investigação, relatando maior prevalência de tratamento entre os mais velhos ${ }^{27}$. A ocorrência simultânea de hábitos pouco saudáveis como o tabagismo, sedentarismo e consumo de álcool com a ausência de tratamento foi relatada também em outras investigações ${ }^{26,27}$. Como exceção a este padrão de "aglomeração" de comportamentos saudáveis, maior proporção de hipertensos com sobrepeso ou obesidade, entre os bancários, estava em tratamento, o que também foi encontrado entre os norte-americanos ${ }^{26}$. Este resultado pode ser consequiência da maior insistência na aderência ao tratamento por parte dos médicos ou do acompanhamento mais freqüente dos níveis tensionais nos pacientes obesos, em função da presença desta condição. Situação semelhante pode justificar a maior proporção de hipertensos em tratamento entre aqueles que apresentavam história familiar de doenças cerebrovasculares, embora o mesmo fosse esperado naqueles com história familiar de hipertensão ou IAM, o que não foi encontrado.

Limitações relacionadas ao delineamento transversal do estudo não permitem afirmações sobre mudanças de hábitos e comportamentos após o diagnóstico de HA. É possível, no entanto, relatar a situação da população de estudo no momento em que a pesquisa foi realizada. A alta prevalência de sobrepeso e obesidade entre os hipertensos pode ser justificada pela importância do excesso de peso como fator de risco para a ocorrência de HA. Com exceção da prática de dieta, os funcionários que "sabiam" ser hipertensos não apresentaram hábitos de vida mais saudáveis do que os não hipertensos: não houve diferenças importantes entre hipertensos e não hipertensos em relação ao consumo de álcool ou prática de atividades físicas regulares, enquanto o hábito de fumar foi mais frequiente entre os hipertensos jovens do que entre os não hipertensos.

A disponibilidade de assistência à saúde e acesso à informação não foram suficientes, no caso dos bancários, para garantir o tratamento nem tampouco a adoção de hábitos e comportamentos que contribuem para o controle dos níveis de PA. Diversos estudos têm demonstrado que educação, informação e acesso a bens e serviços não são suficientes para causar e manter mudanças de hábitos e atitudes relacionadas à saúde ${ }^{30-32}$. Circunstâncias de vida, pessoais e sociais, como situação conjugal, escolaridade e idade, conforme resultados deste estudo, e também as percepções do indivíduo sobre seu próprio bem estar, fazem parte desta rede complexa de fatores que condicionam atitudes relacionadas à saúde. Além disso, o valor social atribuído a determinados hábitos é também de grande importância, principalmente entre os jovens. Viver em uma sociedade onde alertas sobre riscos de doença convivem com estímulos para o con- sumo de tabaco, álcool e comida industrializada, cria um ambiente onde duplas mensagens têm que ser processadas, na maior parte do tempo ${ }^{33}$. Doenças crônicas, que se desenvolvem de forma insidiosa e podem apresentar ou não conseqüências graves a longo prazo, acabam perdendo a disputa no raciocínio de custo-benefício que fazemos ao escolher determinado comportamento. Dessa maneira, é preciso aceitar o caráter não racional, pelo menos do ponto de vista médico, das escolhas de comportamento, especialmente quando se trata do prazer, seja ele ligado ao hábito de fumar, beber, comer ou à vida sexual ${ }^{33}$.

Outro aspecto a considerar entre as possíveis justificativas para a baixa prevalência de hipertensos em tratamento é a dificuldade de convencer o paciente, muitas vezes assintomático, de que ele é doente, especialmente quando este rótulo implica na mudança de hábitos prazerosos ou ainda na obrigação de usar medicamentos para sempre. De fato, esta classificação em doentes e não doentes é o resultado da aplicação de pontos de corte arbitrários numa escala que é contínua ${ }^{34,35}$. Nas populações ocidentais, praticamente todos os indivíduos são portadores de algum grau de HA e doença coronária, sendo a diferença de caráter quantitativo e não qualitativo (doentes x não doentes). Não foi possível, por exemplo, identificar um limiar crítico dos principais fatores de risco, abaixo do qual o risco de doença não existe. O que foi possível demonstrar é que, quanto mais baixos os níveis de PA e quantidade de cigarros consumidos, por exemplo, menor o risco de doenças cardiovasculares, a longo prazo ${ }^{36,37}$. Assim, se em muitos casos os níveis de PA não deixam dúvidas e demandam o início imediato do tratamento farmacológico, em outros, apenas a mudança de hábitos, que interferem nos níveis de PA, devem ser aconselhados, não só aos possíveis hipertensos, mas ao conjunto da população.

Muito há ainda a fazer para o controle da HA em nosso país. Desde garantir o acesso de toda a população aos serviços de saúde, onde a medida da PA deve fazer parte da consulta, seja qual for a queixa do paciente, até a implantação de políticas de saúde que incentivem mudanças de hábitos e comportamentos relacionados à saúde. Quanto ao atendimento individual, os inúmeros relatos de abandono do tratamento anti-hipertensivo chamam a atenção para o fracasso da abordagem de "fazer cumprir uma ordem médica" 30,38,39. Todas as dimensões envolvidas nas escolhas de comportamento e na decisão de aderir a tratamentos, além da noção de que o conhecimento científico está em contínua modificação, devem contribuir para o desenvolvimento da relação médicopaciente cada vez mais aberta, franca e cooperativa.

\section{Agradecimentos}

Ao Prof. Eduardo Faerstein, pelas valiosas sugestões ao artigo original; Prof. Ruy Laurenti, pelas sugestões a pesquisa; Prof. Milena P. Duchiade, Maria de Jesus M. Fonseca, Célia Regina de Andrade, Kaizô Beltrão, Joaquim Valente, Carlos H. Klein, José Lozana e Margareth Portela, pelo auxílio em várias etapas da pesquisa. 


\section{Referências}

1. Keys A - Coronary heart disease in seven countries. Circulation.1970; 41(suppl I): 1-211.

2. Hypertension Detection and Follow-up Group - The effect of treatment on mortality in "mild" hypertension. N Engl J Med 1982; 307: 976-80.

3. Klein CH, Silva NAS, Nogueira AR, Campos LHS, Bloch KV - Hipertensão arterial na Ilha do Governador - RJ. Relatório de Pesquisa (mimeo), 1992.

4. Duncan BB, Schmidt MI, Polanczyk CA, Homrich CS, Rosa RR, Achutti AA Fatores de risco para doenças não transmissíveis em área metropolitana da região Sul do Brasil. Prevalência e simultaneidade. Rev. Saúde Pública 1993; 27: 43-8.

5. Lolio CA - Prevalência da hipertensão arterial em Araraquara. Arq Bras Cardiol 1990; 55: 167-73.

6. The Sixth Report of the Joint National Committee on Prevention, Detection, Evaluation, and Treatment of High Blood Pressure. Arch Intern Med 1997; 157: 2413-45.

7. Casper M, Wing S, Strogatz D, Davis CE, Tyroler, HA - Antihypertensive treatment and US trends in stroke mortality, 1962 to 1980 . Am J Public Health 1992; 82: 1600-6.

8. Grupo Técnico CASSI-Pesquisas Epidemiológicas - Condições de Saúde dos Associados da Caixa de Assistência dos Funcionários do Banco do Brasil. Relatório de Pesquisa, 1996.

9. Chor D - Perfil de risco cardiovascular em funcionários de banco estatal. Tese de Doutoramento. Faculdade de Saúde Pública da USP. São Paulo, 1997.

10. Chor D, Coutinho ESF, Laurenti R - Condições de saúde de funcionários de banco estatal no Rio de Janeiro: confiabilidade da informação de peso e estatura. Rev Saúde Pub 1999; 33 (no prelo).

11. Rose G - Standardization of observers in blood pressure measurement. Lancet 1965; $27: 673-4$

12. Habicht JP - Standardización de métodos epidemiológicos quantitativos sobre el terrén. Boletin de la Oficina Sanitaria Panamericana, 1978; 76: 375-81.

13. Organização Mundial da Saúde - Hipertensão Arterial. Série de Informes Técnicos, 1978; 628: 61p

14. Gray DS - Diagnosis and prevalence of obesity. Med Clin North America 1989; 73: $15.1-13$

15. Fletcher RH, Fletcher SW, Wagner EH - Clinical Epidemiology. The essentials New York: Williams \& Wilkins, 1996: 43-74.

16. Fleiss JL - Statistical Methods for Rates and Proportions. New York: John Wiley, 1981: 218-20.

17. Shrout PE, Fleiss JL - Intraclass correlations: uses in assessing rater reliability. Psychological Bulletin 1979; 86: 420-8

18. Norusis MS, SPSS for windows - Base system user's guide release 5.0. Chicago: SPSS Inc, 1992.

19. US Department of Health and Human Services - Office of Disease Prevention and Health Promotion. Developing objectives for healthy people 2010, 1997.

20. Organización Mundial dela Salud. Organización Panamericana de la Salud Programa de Intervención Integrada de Alcance Nacional en Enfermedades no Transmisibles (CARMEN/CINDI) - Protocolo y Directrices, 1997.
21. Klein CH - Hipertensão arterial nos estratos geo-econômicos do Rio Grande do Sul. (Tese de Mestrado). Rio de Janeiro: Escola Nacional de Saúde Pública/ FIOCRUZ, 1984

22. Klein CH, Araújo JWG, Leal MC - Inquérito epidemiológico sobre hipertensão arterial em Volta Redonda - RJ. Cad Saúde Pública 1985; 1: 58-70.

23. Monteiro CA, Mondini L, Souza ALM, Popkin BM - The nutrition transition in Brazil. Eur J Clin Nutr 1995; 49: 105-13.

24. Rego R, Berardo ANF, Rodrigues SSR et al - Fatores de risco para doença crônicas não transmissíveis: inquérito domiciliar no município de São Paulo, SP (Brasil). Metodologia e resultados preliminares. Rev Saúde Pública 1990; 24: 277-85.

25. Brownson RC, Jackson-Thompson J, Wilkerson JC, Kiani F - Reliability of information on chronic disease risk factors collected in the Missouri Behavioral Risk Factor Surveillance System. Epidemiology 1994; 5: 545-9.

26. Nieto FJ, Alonso J, Chambless LE et al - Population awareness and control of hypertension and hypercholesterolemia. The Atherosclerosis Risk in Communities Study. Arch Intem Med 1995; 155: 677-84.

27. Joffres MR, Hamet $P$, Rabkin, $S W$ et al - Prevalence, control and awareness of high blood presure among canadian adults. Can Med Assoc J 1992; 146: 1997-2005

28. Fuchs FD, Moreira LB, Moraes RS, Bredemeier M, Cardozo SC - Prevalência de hipertensão arterial sistêmica e fatores associados na região urbana de Porto Alegre. Estudo de base populacional. Arq Bras Cardiol 1995; 63: 473-9.

29. Clark LT - Improving compliance and increasing control of hypertension: needs of special hypertensive populations. Am Heart J 1991; 121: 664-9.

30. Lessa I, Fonseca J - Raça, aderência ao tratamento e/ou consultas e controle da hipertensão arterial. Arq Bras Cardiol 1997; 68: 443-9.

31. Rose G - The Strategy of Preventive Medicine. New York: Oxford University Press, 1992: 64-135.

32. Marmot MG - From alcohol and breast cancer to beef and BSE - Improving our communication of risk. Am J Public Health 1996; 86: 921-3.

33. Castiel LD - Força e vontade: aspectos teórico-metodológicos do risco em epidemiologia e prevenção de HIV/AIDS. Rev Saúde Pública 1996; 30: 91-100.

34. Pickering G - Normotension and hypertension: the mysterious viability of the false. Am J Med 1978; 65: 561-3.

35. Rose G - Strategies of prevention: the individual and the population. In: Marmot M, Elliott P (orgs) - Coronary Heart Disease Epidemiology - From Aetiology to Public Health. New York: Oxford University Press, 1992: 311-24.

36. Rose G, Shipley M - Plasma cholesterol concentration and death from coronary heart disease: 10 year results of the Whitehall study. Br Med J 1986; 293: 306-7.

37. Meisler JG, St Jeor S - Foreword. Am J Clin Nutr 1996; 63: 4095-115.

38. Florezano RU, Roessler EB, Scharazer JZ, Soto IZ, Cantú AM - Permanencia en tratamiento antihipertensivo: comparacion de dos sistemas de seguimiento. Bol Of Panam 1981; 91: 428-40

39. Donovan JL, Blake DR - Patient non-compliance: deviance or reasoned decision-making? Soc Sci Med 1992; 34: 507-13. 